\title{
A novel biodegradable self-healing hydrogel to induce blood capillary formation
}

\author{
Fu-Yu Hsieh ${ }^{1}$, Lei Tao ${ }^{2,3}$, Yen Wei ${ }^{2,3}$ and Shan-hui Hsu ${ }^{1,4,5}$ \\ A major challenge in tissue engineering is to generate a functional microvasculature that ensures proper blood perfusion and \\ connection with surrounding tissues. Strategies such as the incorporation of growth factors have been proposed to induce the \\ growth of new blood vessels into engineered tissue, but limitations remain. Herein a novel chitosan-fibrin (CF)-based self-healing \\ hydrogel with a modulus of $\sim 1.2 \mathrm{kPa}$ was developed. The self-healing hydrogel was found to be injectable and to degrade \\ $\sim 70 \%$ in 2 weeks. Vascular endothelial cells seeded in the CF hydrogel were able to form capillary-like structures. Moreover, the \\ injection of the CF hydrogel alone promoted angiogenesis in the perivitelline space of zebrafish and rescued the blood circulation \\ in ischemic hindlimbs of mice. The excellent self-healing and angiogenic capacities of the hydrogel may be associated with the \\ formation of an interpenetrating polymer network structure between chitosan and fibrin. This unique self-healing hydrogel offers \\ new possibilities for future applications to vascular repair.
}

NPG Asia Materials (2017) 9, e363; doi:10.1038/am.2017.23; published online 17 March 2017

\section{INTRODUCTION}

Hydrogels are attractive materials for biomedical applications because of their high water content. ${ }^{1}$ Their favorable nutrient and oxygen transport has made them suitable for cell culture and tissue engineering. A major limitation for engineered tissues is the lack of blood vessels. One of the strategies to promote vascularization is to incorporate vascular endothelial growth factors (VEGFs) into the hydrogels or scaffolds. ${ }^{2}$ Smart hydrogels are promising growth factor and cell delivery vehicles for therapeutic healing and tissue regeneration because of their responsiveness to various environmental stimuli, such as solvent composition, temperature, $\mathrm{pH}$, electric field and light. Injectable hydrogels are especially appealing because they provide possibilities for minimally invasive delivery procedures. ${ }^{3,4}$ Self-healing hydrogels are a specialized type of hydrogel that can autonomously repair cracks or focal damage and their integrity as well as their mechanical properties can recover after being destroyed. ${ }^{5,6}$ Recently, self-healing hydrogels have been developed from structurally dynamic polymers with dynamic covalent chemistry. ${ }^{7-9}$ The dynamic selfhealing hydrogel with reversible crosslinkages have been shown to mimic the rheological properties of physiological fluids, such as the synovial fluid. ${ }^{7}$ Self-healing hydrogels based on dynamic covalent chemistry thus hold promise for biomedical applications.

Although self-healing is one of the fundamental properties of living tissues, biomedical applications of self-healing materials have seldom been reported. Most of the literature has focused on the synthesis and self-healing mechanism of non-biodegradable self-healing materials as well as their applications in various industrial fields, such as electronics, automotive and chemical production. ${ }^{10}$ Nevertheless, there have been several reports on the applications of biodegradable self-healing materials as drug or cell delivery carriers. ${ }^{11-14}$ In a recent study, a nanofibrous self-healing hydrogel consisting of multidomain peptides has been incorporated with angiogenic signaling proteins to promote the growth of blood vessel networks into the hydrogel. ${ }^{15}$ To date, no self-healing hydrogel has been demonstrated to have intrinsic structural properties that induce angiogenesis and capillary formation. In the current study, we present a novel injectable, gradually degrading self-healing hydrogel that induces blood capillary formation in vitro and in vivo.

Chitosan (CS) is a natural linear cationic polymer of glucosamine and $\mathrm{N}$-acetyl glucodamine, and it has been widely used in the pharmaceutical and tissue engineering fields, owing to its biocompatibility and biodegradability. Several reports have indicated that CS as a biomaterial promotes angiogenesis in corneal and skin tissues. ${ }^{16,17}$ Moreover, other studies have reported that CS-based hydrogels do not show any angiogenic potential in vitro and in vivo. ${ }^{18}$ Fibrin is a biological polymer in vertebrates that maintains arterial endothelial cell (EC) adherence and induces angiogenesis at the site of injury. Fibrin gel is utilized in many in vitro models of angiogenesis and vasculogenesis. However, fibrin gel suffers from the drawbacks of mechanical weakness and instability ${ }^{19}$ and is not suitable for tissue

\footnotetext{
${ }^{1}$ Institute of Polymer Science and Engineering, National Taiwan University, Taiwan, R.O.C.; ${ }^{2}$ Department of Chemistry, Tsinghua University, Beijing, P.R. China; ${ }^{3}$ Key Lab of Organic Optoelectronic \& Molecular Engineering of Ministry of Education, Tsinghua University, Beijing, P.R. China; ${ }^{4}$ Research and Development Center for Medical Devices, National Taiwan University, Taiwan, R.O.C. and ${ }^{5}$ Institute of Cellular and System Medicine, National Health Research Institutes, Taiwan, R.O.C.

Correspondence: Professor S-h Hsu, Institute of Polymer Science and Engineering, National Taiwan University, No. 1, Sec. 4, Roosevelt Road, Taipei 10617, Taiwan, R.O.C. E-mail: shhsu@ntu.edu.tw

or Professor Y Wei, The Key Laboratory of Bioorganic Phosphorus Chemistry \& Chemical Biology, Department of Chemistry, Tsinghua University, Beijing 100084, P.R. China.

E-mail: weiyen@tsinghua.edu.cn

Received 20 August 2016; revised 29 October 2016; accepted 28 December 2016
} 
engineering applications. Furthermore, fibrin gel cannot be injected after it has formed. A self-healing gel based on fibrin has not been reported to date. In this study, we hypothesized that a composite self-healing hydrogel based on CS and fibrin might overcome the limitation of each single component. This newly developed hydrogel was characterized for its self-healing properties and its ability to induce blood capillary formation.

\section{MATERIALS AND METHODS}

\section{Preparation and characterization of the different hydrogels}

Synthesis of telechelic difunctional poly(ethylene glycol) (DF-PEG) was first achieved via esterification of hydroxyl-terminated PEG with 4-formylbenzoic acid. $^{20}$ The DF-PEG solution was prepared by dissolving the synthesized polymer in distilled and deionized water. The glycol CS solution was prepared by dissolving the glycol CS powder (Sigma, St Louis, MO, USA) in distilled and deionized water. Fibrinogen (Sigma) was dissolved in distilled and deionized water to obtain a fibrinogen solution. Thrombin (Sigma) was dissolved in distilled and deionized water to obtain a thrombin solution. The gel modulus of the chitosan-fibrin (CF) and CS hydrogels was tuned by the concentrations of each component. The CF hydrogel was achieved by mixing the DF-PEG solution, glycol CS solution, fibrinogen solution and thrombin solution. The concentrations of each component were optimized for encapsulating cells in the current study. The appropriate formula of the CF hydrogel was found to contain $1.5 \%$ glycol CS, $1 \%$ DF-PEG, $0.005 \%$ fibrinogen and $0.002 \%$ thrombin in the final gel. For comparison, CS hydrogel of the same stiffness was also used, which contained $1.5 \%$ glycol CS and 1\% DF-PEG in the final gel. Fibrin gel served as the positive control and consisted of $0.5 \%$ fibrinogen and $0.2 \%$ thrombin. The formula of the fibrin gel has been widely used for the in vitro endothelial tube formation assay. Other groups of hydrogels include CS-RGD hydrogel (1.5\% glycol CS, $1 \%$ DF-PEG and 5\% arginyl-glycyl-aspartic acid (RGD) protein $\left.{ }^{21}\right)$, CS-fibrinogen hydrogel (1.5\% glycol CS, $1 \%$ DF-PEG and $0.005 \%$ fibrinogen) and CS-thrombin hydrogel (1.5\% glycol CS, $1 \%$ DF-PEG and $0.002 \%$ thrombin).

The shear strain-dependent viscoelastic properties of the hydrogels were measured with a rheometer (HR-2, TA Instruments, New Castle, DE, USA) with a cone and plate geometry at a frequency of $1 \mathrm{~Hz}$. For the CS hydrogels, $3 \%$ CS solution $(0.2 \mathrm{ml})$ was first spread on the plate. Then $2 \%$ DF-PEG solution $(0.2 \mathrm{ml})$ was added onto the CS solution and mixed by pipetting. For the CF hydrogels, $6 \%$ CS solution $(0.1 \mathrm{ml})$ was first spread on the plate, and this was followed by $0.02 \%$ fibrinogen solution $(0.1 \mathrm{ml}), 2 \%$ DF-PEG solution $(0.1 \mathrm{ml})$ and $0.008 \%$ thrombin solution $(0.1 \mathrm{ml})$, which were mixed by pipetting. The temperature was adjusted to $37^{\circ} \mathrm{C}$ during the measurement. For time-dependent measurement, the storage and loss moduli were measured against time at the constant frequency of $1 \mathrm{~Hz}$ and strain of $1 \%$. To measure the fibrin gel, a $10 \%$ fibrinogen solution $(0.2 \mathrm{ml})$ was first loaded onto the plate. Then a $4 \%$ thrombin solution $(0.2 \mathrm{ml})$ was dropped onto the fibrinogen solution. In another set of experiments, the gel was subjected to the strain sweep test at $1 \mathrm{~Hz}$ while the strain was increased from 1 to $400 \%$. The self-healing properties were also evaluated via rheology. Continuous step changes in the oscillatory strain between 150 and $1 \%$ under the same frequency $(1 \mathrm{~Hz})$ were applied to test the strain-induced destruction and recovery of the hydrogel. Shear thinning was induced via application of $150 \%$ strain for $10 \mathrm{~min}$. The strain was then decreased to $1 \%$ for $10 \mathrm{~min}$ to allow the gel to recover.

\section{Thermal analysis}

For thermogravimetric analysis, the decomposition profile of the dried samples (CS, CF and CF' hydrogels) was thermogravimetrically analyzed (Q50, TA Instrument, New Castle, DE, USA). Dried samples that ranged from 4 to $6 \mathrm{mg}$ were placed in the pan and heated from 100 to $800^{\circ} \mathrm{C}$ under a nitrogen atmosphere at a heating rate of $10^{\circ} \mathrm{C} \mathrm{min}^{-1}$, and the weight loss was recorded as a function of temperature. Differential scanning calorimetry (DSC 2500, TA Universal) was performed to measure the crystallinity and melting point of the dried samples (CS, CF and CF' hydrogels). Each sample was heated from 70 to $130{ }^{\circ} \mathrm{C}$, with a heating rate of $10^{\circ} \mathrm{C} \mathrm{min}^{-1}$ under nitrogen purging.
The pan and lid were aluminum. The samples weighed approximately $5 \mathrm{mg}$ for each measurement.

\section{In vitro biodegradation}

Hydrogels that weighed $50 \mathrm{mg}$ were placed in $5 \mathrm{ml}$ of a phosphate-buffered saline (PBS) solution ( $\mathrm{pH} 7.4$ ). The hydrogels were incubated at $37^{\circ} \mathrm{C}$ for 5,10 , 20 and 30 days. The percentage residual mass (\%) of the hydrogels was calculated from the following equation:

Residualmass $(\%)=\left(W_{t} / W_{0}\right) \times 100 \%$,

where $W_{0}$ is the initial weight of the hydrogel and $W_{t}$ is the weight of the hydrogel at each time point.

\section{Culture of vascular endothelial cells}

Adult bovine carotid artery ECs were obtained by collagenase digestion. ${ }^{22}$ ECs were cultured in Dulbecco's modified Eagle's medium (DMEM; Gibco, Invitrogen, Carlsbad, CA, USA) supplemented with $10 \%$ fetal bovine serum (Gibco, Invitrogen) and $100 \mathrm{U} \mathrm{ml}^{-1}$ penicillin-streptomycin (Caisson Labs, Smithfield, UT, USA). The cultures were maintained in $5 \% \mathrm{CO}_{2}$ and a $37{ }^{\circ} \mathrm{C}$ incubator. The culture medium was refreshed every 2 days.

\section{Cell labeling}

ECs were labeled with a red fluorescent dye (PKH26 Red Fluorescence Cell Linker Kit, Sigma) in vitro. The cells (a density of $1 \times 10^{7}$ cells $\mathrm{ml}^{-1}$ ) were labeled by mixture with $2 \times 10^{-6} \mathrm{M}$ PKH26, which stably incorporates into the cell membrane, owing to its long aliphatic tails. The labeling process was stopped with complete medium. The labeled cells were washed and ready for use.

\section{In vitro angiogenesis assay for cells encapsulated in various hydrogels}

The PKH26-labeled ECs were embedded by mixture of cells with each hydrogel, before gelation, to achieve $1 \times 10^{6}$ cells in a 1-ml volume of hydrogel. The cell-laden hydrogels were immediately placed in an incubator. The culture medium was refreshed after 2 days. After incubation at $37^{\circ} \mathrm{C}$ for 3 days, images of six random microscopic fields were taken for each hydrogel. The mean branching length and branch point density were determined with the ImageJ software (National Institutes of Health, Bethesda, MD, USA). These data served as indices of the capillary-forming ability.

\section{Gene expression for cells encapsulated in hydrogels}

To determine the vascularization levels of ECs encapsulated in hydrogels, the expression levels of vascular endothelial-related genes was analyzed using real-time reverse transcriptase-PCR. Total RNA was extracted with TRIzol reagent (Invitrogen, Carlsbad, CA, USA) from cell-laden hydrogels at 3 days. RNA was reverse transcribed into cDNA and amplified with a RevertAid First Strand cDNA Synthesis Kit (MBI Fermentas, St. Leon-Rot, Germany). Realtime reverse transcriptase-PCR was performed using the DyNAmo Flash SYBR Green qPCR Kit (Finnzymes Oy, Espoo, Finland). The PCR reactions and detection were performed with an Applied Biosystems StepOne instrument (Foster City, CA, USA). The expression was normalized to that of GAPDH (glyceraldehyde 3-phosphate dehydrogenase). The primer sequences for each gene used in this study are shown in Supplementary Table S1.

\section{Metabolic flux analysis by Seahorse XF-96}

The oxygen consumption rate and extracellular acidification rate were measured at $37^{\circ} \mathrm{C}$ using an XF96 extracellular analyzer (Seahorse Bioscience, Billerica, MA, USA). ECs in culture medium were mixed with each hydrogel such that the final mixture contained $1 \times 10^{7} \mathrm{cells} \mathrm{ml}^{-1}$. The EC-embedded hydrogel $(5 \mu \mathrm{l}$ per well) was placed into eight-well plates and incubated in the incubator for $24 \mathrm{~h}$. The medium was changed to unbuffered DMEM (DMEM supplemented with $25 \mathrm{~mm}$ glucose, $1 \mathrm{~mm}$ sodium pyruvate, $31 \mathrm{~mm} \mathrm{NaCl}$ and $2 \mathrm{~mm}$ GlutaMax, $\mathrm{pH} 7.4$ ), and the hydrogel was incubated at $37^{\circ} \mathrm{C}$ without $5 \%$ $\mathrm{CO}_{2}$ for $1 \mathrm{~h}$. All of the injection reagents were adjusted to $\mathrm{pH} 7.4$ on the day of the assay. The oxygen consumption rate and extracellular acidification rate were 
a

Glycol chitosan
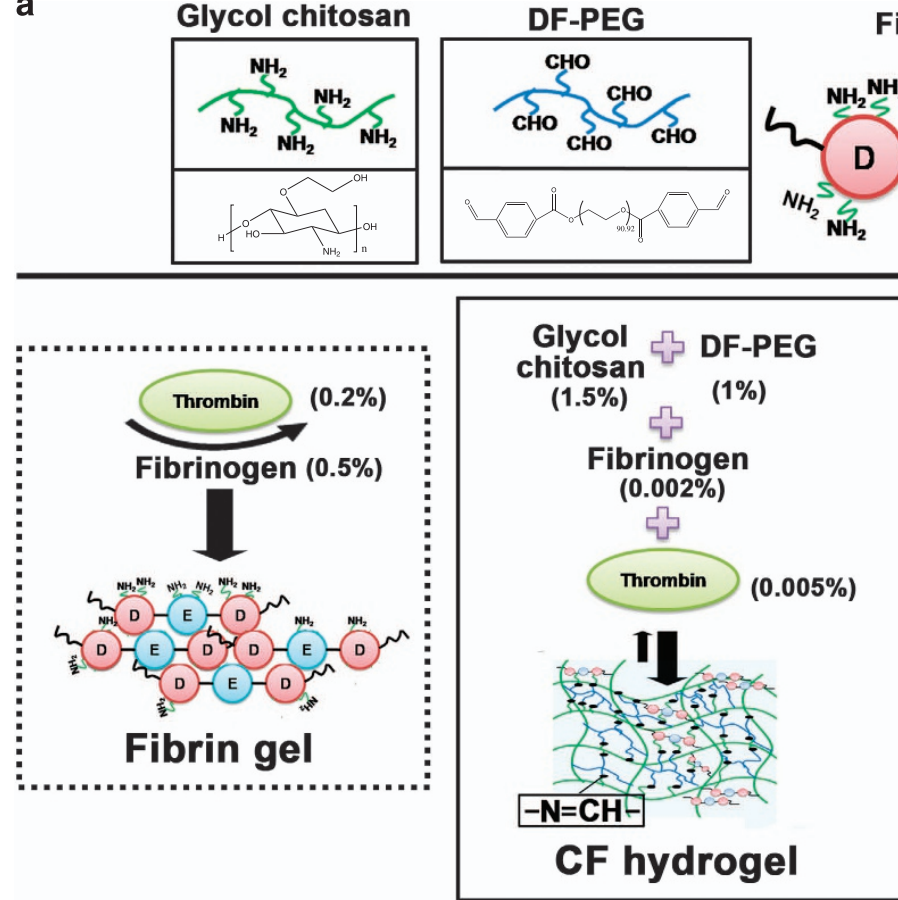

Fibrinogen

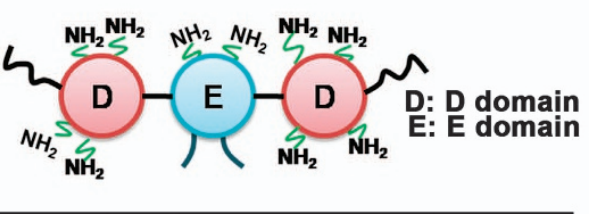

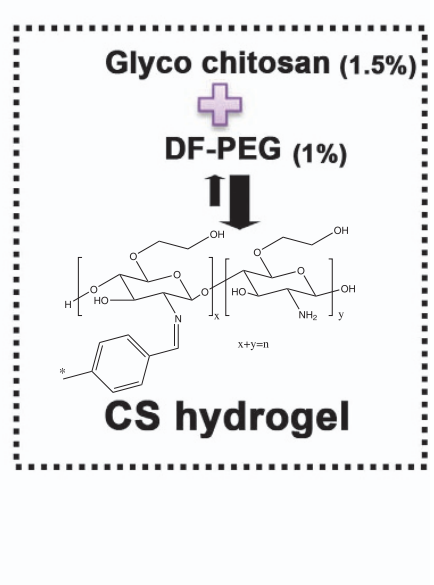

b
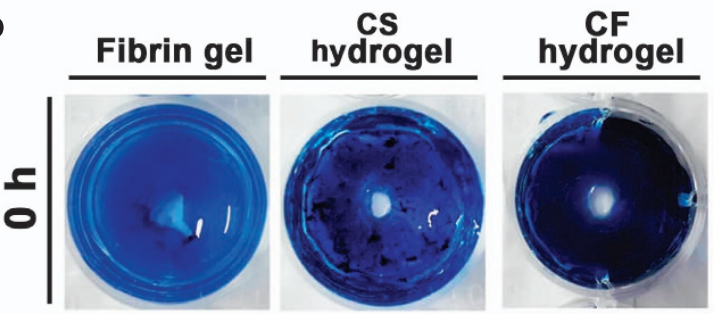

C Fibrin gel
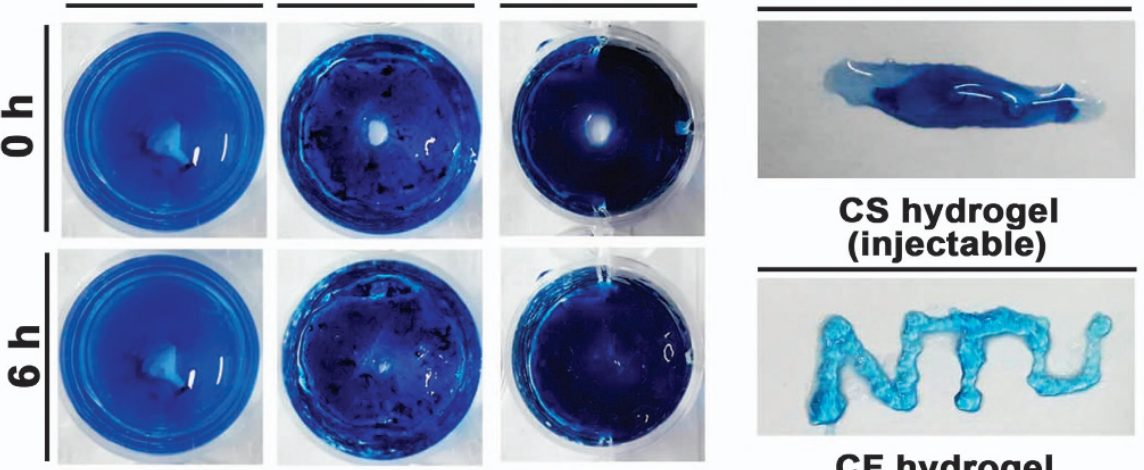

CS hydrogel (injectable)
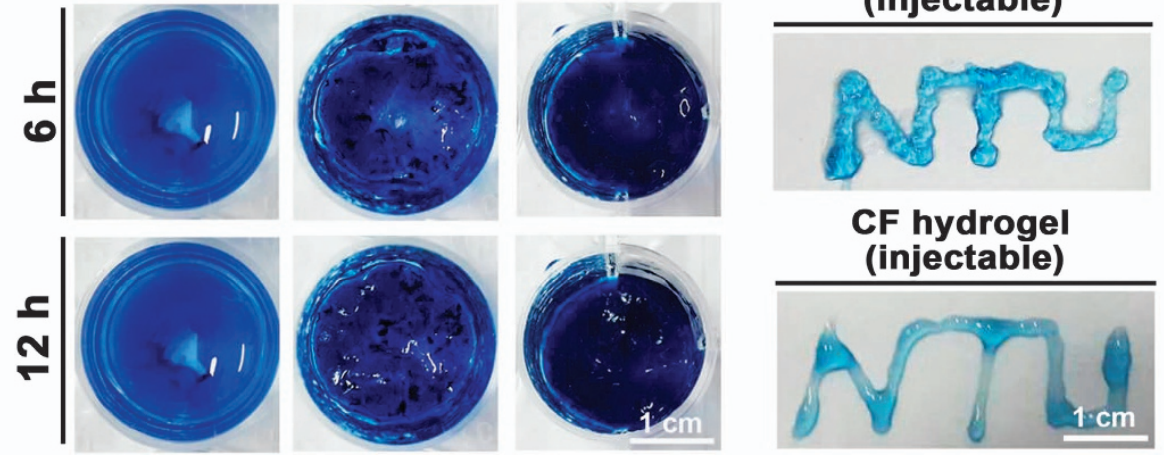

Figure 1 Preparation of the CF hydrogel. (a) Benzaldehydes at both ends of difunctionalized PEG (DF-PEG) crosslinking with glycol chitosan and fibrinogen to form the CF hydrogel. The gel modulus of the self-healing hydrogel was tuned on the basis of the concentrations of each component. The CS hydrogel (without fibrin) and fibrin gel (without the CS component) were also prepared for comparison. (b) Gross appearance of the self-healing process of various hydrogels. (c) The CF and CS hydrogels were able to pass through a 26 -gauge $(457 \mu \mathrm{m})$ needle without clogging. Fibrin gel was able to pass through only a cylindrical syringe (2-mm inner diameter). The gels were stained with trypan blue for observation.

automatically calculated with the Seahorse XF-96 software. Every point represents an average of 3-6 different wells.

\section{Zebrafish experiment}

$\mathrm{Tg}$ (fli1a:EGFP) zebrafish embryos were purchased from the Zebrafish International Resource Center (ZIRC, Eugene, OR, USA) and were raised, maintained and paired under standard conditions. ${ }^{23}$ The flila promoter-driven enhanced green fluorescent protein (EGFP) is predominantly expressed in vascular endothelial and endocardial cells. Zebrafish were maintained in Petri dishes with
E3 embryo medium $(5 \mathrm{~mm} \mathrm{NaCl}, 0.17 \mathrm{~mm} \mathrm{KCl}, 0.33 \mathrm{~mm} \mathrm{CaCl} 2$ and $0.33 \mathrm{~mm}$ $\left.\mathrm{MgSO}_{4}\right)$ at $28{ }^{\circ} \mathrm{C}$. Animals were divided into three groups in this study ( $n=90$ per group). The first group comprised zebrafish embryos injected with PBS at $24 \mathrm{~h}$ postfertilization (hpf) as a blank control. The other two groups were injected with CF hydrogel or CS hydrogel at $24 \mathrm{hpf}$. The injection sites were the subintestinal vessels of the zebrafish embryos. ${ }^{24}$ The subintestinal vessel pattern in $\mathrm{Tg}$ (flila:EGFP) zebrafish embryos was evaluated at $48 \mathrm{hpf}$. The number of subintestinal vessel branching points in $48 \mathrm{hpf}$ zebrafish embryos was quantified for each group. ${ }^{23}$ The number of subintestinal vessel branching points is an index of the capillary-forming ability. The studies were repeated two additional times. 
a

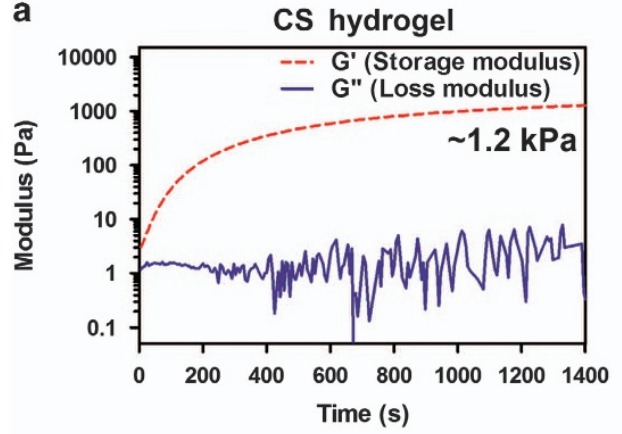

C

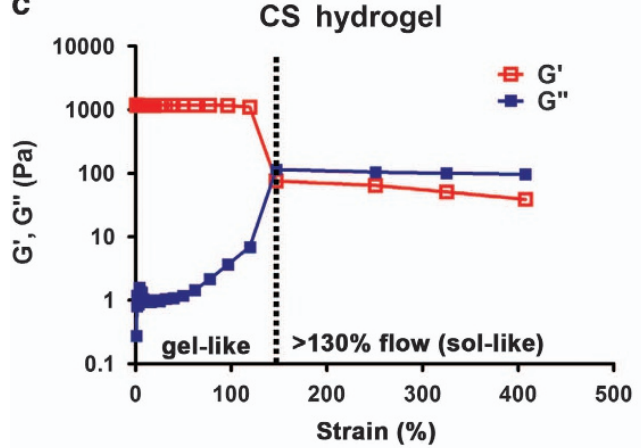

e

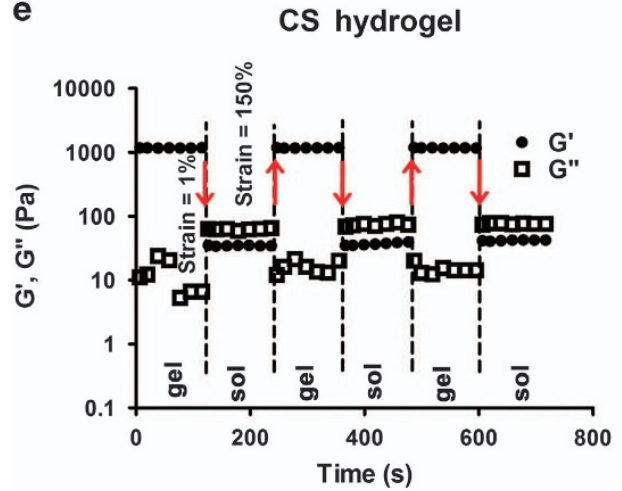

b

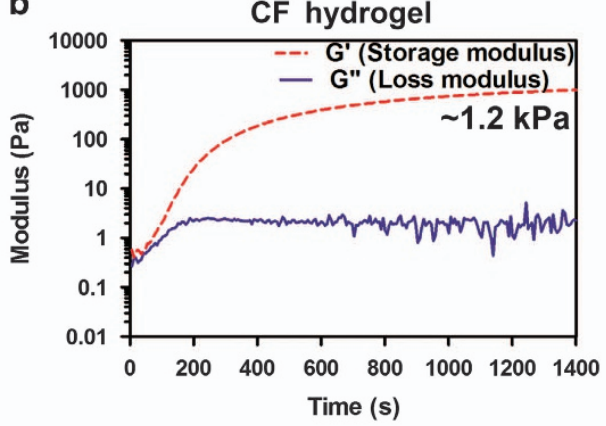

d

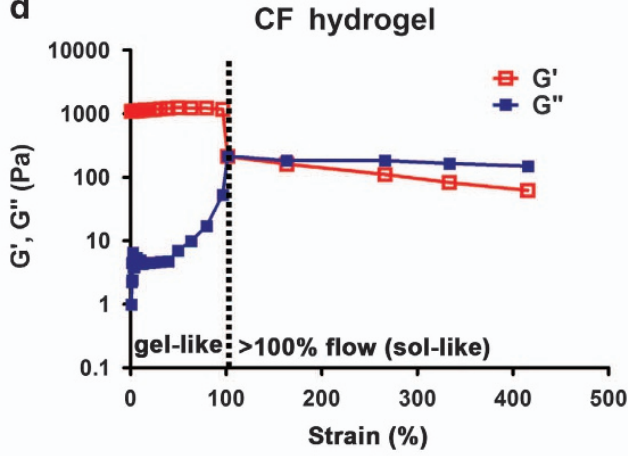

$\mathbf{f}$

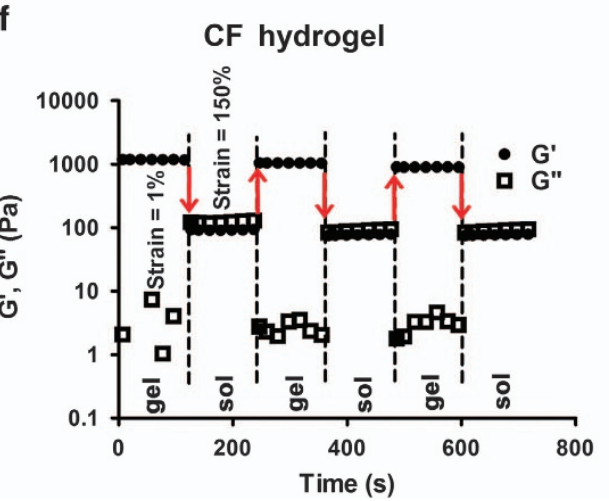

Figure 2 Rheological properties of CF hydrogel vs CS hydrogel. (a, b) The gelation time and modulus $\left(G^{\prime}\right.$ and $\left.G^{\prime \prime}\right)$ of the (a) $\mathrm{CS}$ and (b) $\mathrm{CF}$ hydrogels at $37^{\circ} \mathrm{C}$ $\left(1 \%\right.$ strain and $1 \mathrm{~Hz}$ ). (c, d) The strain sweep curves for the (c) CS and (d) CF hydrogels at $37^{\circ} \mathrm{C}$ and $1 \mathrm{~Hz}$. (e, f) The damage-healing properties of the (e) CS and (f) $\mathrm{CF}$ hydrogels demonstrated by the continuous step strain ( $1 \%$ strain $\rightarrow 150 \%$ strain $\rightarrow 1 \%$ strain) measurements at $37^{\circ} \mathrm{C}$ and $1 \mathrm{~Hz}$.

Mouse model of hindlimb ischemia and treatment

The surgical mortality rate was $0 \%$. The left femoral artery and iliac artery in the mice were ligated and then cut to induce hindlimb ischemia. ${ }^{25}$ The CF hydrogel or PBS (control) was intramuscularly injected into the ischemic hind limbs at 2 or 15 days postsurgery. The injections were delivered into two different sites (proximal and distal) at the ischemic area (100 $\mu$ l for each site). Microvascular blood flow was measured with a laser Doppler monitor (VMS-LDF, Moor Instruments, Axminster, UK). The blood flow before operation, 1 day afterward and every week for the following 5 weeks was recorded for both limbs. The data are presented as the blood flow ratio of the ischemic limb (left) to the normal limb (right).

\section{Statistical analysis}

Data from the experiments are presented as mean \pm s.d. For each type of experiment, three similar experiments were performed independently. Statistical differences among the experimental groups were determined by one-way analysis of variance. The results were considered to be statistically significant when the $P$-values were $<0.05$.

\section{RESULTS AND DISCUSSION}

The CF self-healing hydrogel, CS-based self-healing hydrogel and fibrin gel were prepared as summarized in Figure 1a and Supplementary Figure S1 (Supplementary Data). The synthesis of telechelic DF-PEG was first achieved via esterification of hydroxyl-terminated PEG with 4-formylbenzoic acid. ${ }^{20}$ The DF-PEG aqueous solution was prepared by dissolving the synthesized polymer in distilled and deionized water. The aqueous solutions of glycol CS, fibrinogen and thrombin were all prepared in distilled and deionized water. The modulus of the CS as well as CF hydrogel was tuned according to the amount of each component in the hydrogel. The modulus or stiffness of a material has been shown to have a critical role in angiogenesis. ${ }^{26,27} \mathrm{~A}$ previous study has reported that materials with a modulus of $\sim 1 \mathrm{kPa}$ may favor angiogenesis. $^{28}$ In the current study, the concentration of each component was optimized for embedding cells before gelation and achieving appropriate stiffness $(\sim 1.2 \mathrm{kPa})$ to favor blood capillary formation after gelation. The appropriate formula of the CF hydrogel contained $1.5 \%$ glycol CS, $1 \%$ DF-PEG, $0.005 \%$ fibrinogen and 
a
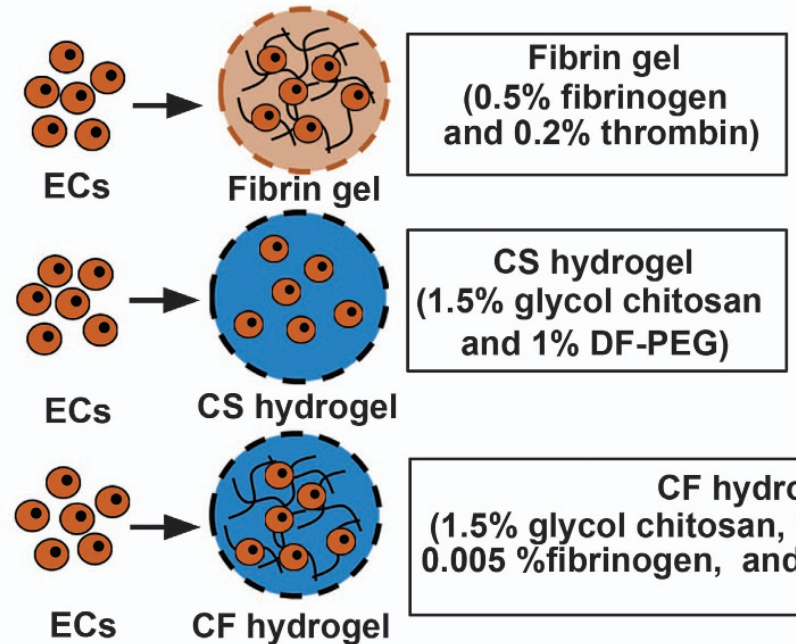

CF hydrogel

(1.5\% glycol chitosan, $1 \%$ DF-PEG, $0.005 \%$ fibrinogen, and $0.002 \%$ thrombin)

b

Fibrin gel

CS hydrogel
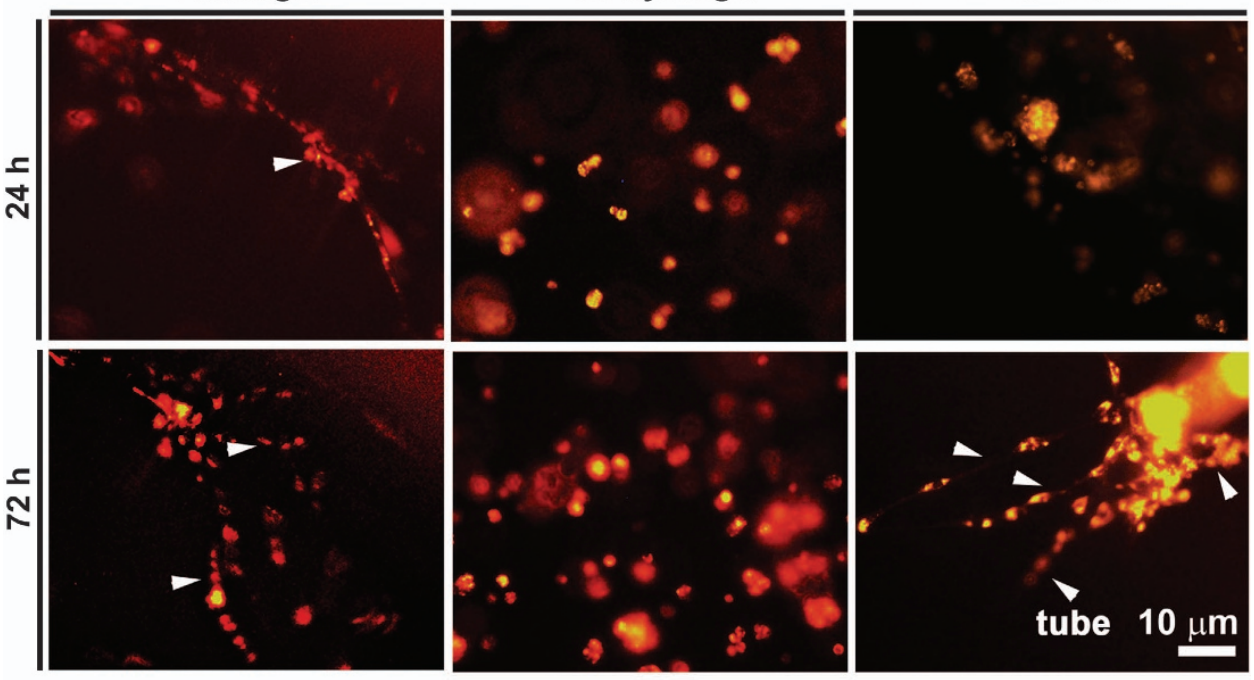

C
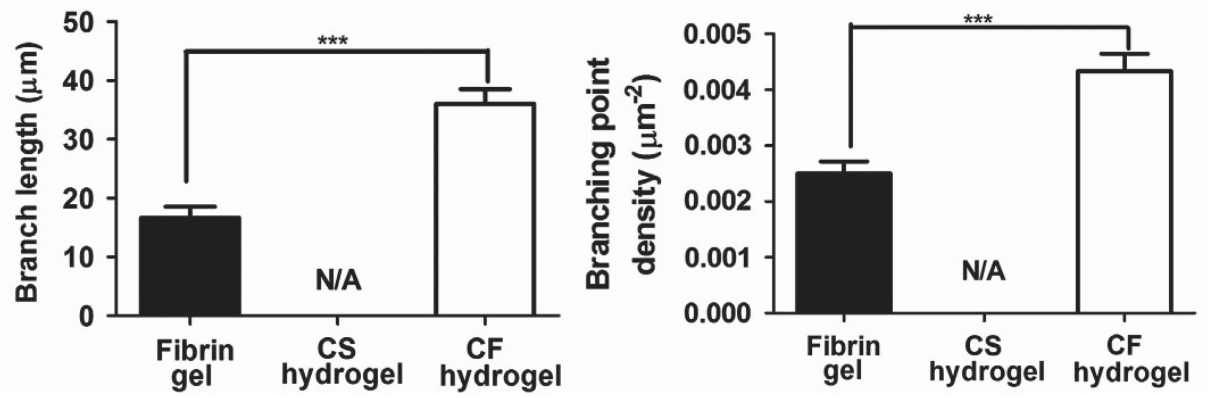

Figure 3 Morphology of vascular endothelial cells (ECs) encapsulated in different hydrogels after injection. (a) Chemical components of the hydrogels. (b) The morphology of the ECs encapsulated in the fibrin gel, CS hydrogel or CF hydrogel after 24 and $72 \mathrm{~h}$. (c) The mean branch length and branching point density of the EC tubes in different hydrogels, which are indices of the capillary-forming ability. The arrowheads indicate $E C$ tubes. $P<0.05, * * P<0.005$, $* * * P<0.001$, among the indicated groups.

$0.002 \%$ thrombin in the final gel. For comparison, CS hydrogel of the same stiffness was used. The proper formula of the CS hydrogel contained $1.5 \%$ glycol CS and 1\% DF-PEG in the final gel. The fibrin gel prepared from $0.5 \%$ fibrinogen and $0.2 \%$ thrombin served as the positive control. This fibrin gel is widely used for the in vitro endothelial tube-formation assay. ${ }^{19}$
The CF hydrogel formed quickly after mixture of the components, through dynamic Schiff-base crosslinkage between the amine groups of CS or fibrinogen and the benzaldehyde groups on the DF-PEG termini (Figure 1a). Because the network was constructed through Schiff-base linkage, the CF hydrogel was expected to be self-healable. To prove that point, a hole was punched in the middle of an $\sim 2.0-\mathrm{cm}$ 
diameter $\mathrm{CF}$ hydrogel (Figure 1b, $0 \mathrm{~h}$ ). The hole was observed to diminish gradually and disappear completely after $\sim 6 \mathrm{~h}$. The self-healed gel looked similar to the initial gel. The CF hydrogel healed automatically without any external stimuli, thus indicating its excellent self-healing capacity. The CS hydrogel, for which self-healing has been reported earlier, ${ }^{14}$ was used for comparison. The central hole in the CS hydrogel shrunk at $6 \mathrm{~h}$, but complete disappearance of the hole occurred after approximately $12 \mathrm{~h}$ (Figure $1 \mathrm{~b}$ ). These results demonstrated that the self-healing process of the CF hydrogel was faster than that of the CS hydrogel. Furthermore, the fibrin gel did not self-heal. Additional self-healing experiments were performed by placing two CF hydrogel strips together to generate one unit of CF hydrogel strip, as illustrated in Supplementary Figure S1c. The results of these experiments confirmed the self-healing of the CS and CF hydrogels but not the fibrin gel. Moreover, both the CS and CF hydrogels were able to be extruded through a 26-gauge needle (diameter: $457 \mu \mathrm{m}$ ) without clogging. In contrast, the fibrin gel was able to pass through only a $2-\mathrm{mm}$ syringe (Figure $1 \mathrm{c}$ ).

The rheological properties of the CS hydrogel, CF hydrogel and fibrin gel were determined with a rheometer (HR-2, TA Instruments). The results are shown in Figure 2 and Supplementary Figure S1. After sample loading, the CS and CF hydrogels underwent rapid gelation at $37^{\circ} \mathrm{C}$ (Figures $2 \mathrm{a}$ and $\mathrm{b}$ ). The storage (elastic) modulus $\left(G^{\prime}\right)$ was close between the two hydrogels, that is, $\sim 1.2 \mathrm{kPa}$ at $37^{\circ} \mathrm{C}$ for both hydrogels. The fibrin gel formed immediately after sample loading, but the gel modulus was relatively low $\left(\sim 0.03-0.04 \mathrm{kPa}\right.$ at $37^{\circ} \mathrm{C}$; Supplementary Figure S1d). The strain-dependent modulus changes in the CS and CF hydrogels (Figures $2 \mathrm{c}$ and $\mathrm{d}$ ) showed differences between the two hydrogels. At $37^{\circ} \mathrm{C}$, the network of the CF hydrogel was destroyed at a strain of $\sim \geqslant 100 \%$. In contrast, the CS hydrogel became sol-like at a strain of $\sim 130 \%$. The damage-healing (sol-gel transition) of the CS and CF hydrogels, which was obtained by application of a large amplitude oscillatory strain ( $\operatorname{strain}=150 \%$, frequency $=1 \mathrm{~Hz}$ ) and recovery at a low strain (strain $=1 \%$, frequency $=1.0 \mathrm{~Hz}$ ), is shown in Figures $2 \mathrm{e}$ and $\mathrm{f}$. The $G^{\prime}$ value of the CS hydrogel decreased from $\sim 1.2$ to $\sim 0.06 \mathrm{kPa}$ when the strain was increased from 1 to $150 \%$ and recovered to $\sim 1.2 \mathrm{kPa}$ when the strain was decreased from 150 to $1 \%$. The self-healing process was also observed for the CF hydrogel: the $G^{\prime}$ decreased from $\sim 1.2$ to $\sim 0.1 \mathrm{kPa}$ and recovered. The quick recovery of the CS and CF inner network confirmed the self-healing capability of the two dynamic hydrogels. The fibrin gel demonstrated no self-healing phenomenon and remained a gel at a $150 \%$ strain (Supplementary Figure S1e).

Degradation of the hydrogels is important in tissue repair. The degradation rates of the CS hydrogel, CF hydrogel and fibrin gel in PBS at $37^{\circ} \mathrm{C}$ are shown in Supplementary Figure S2. The masses of the CS and CF hydrogels gradually decreased by 30 days, whereas the fibrin gel degraded much faster ( $\sim 80 \%$ at 5 days) and fully degraded after only 10 days. The degradation rate of the CF hydrogel was slightly slower than that of the CS hydrogel. Because the formation of the vascular extracellular matrix is a long process in vivo, ${ }^{29}$ we believed that the CF hydrogel, which degraded more slowly, might provide better support for vasculogenesis in vivo.

Arterial ECs were fluorescently labeled with a red fluorescent dye PKH26 and were seeded at a density of $1 \times 10^{6}$ cells ml $^{-1}$ in each hydrogel before gel formation (Figure 3a). The EC-embedded hydrogel was filled in a barrel and extruded through a 26-gauge needle into a Petri dish. The morphologies of the red fluorescence-labeled ECs within various hydrogels were examined under a fluorescence microscope, and the results are shown in Figure 3b. ECs were aggregated in the CS hydrogel at $24 \mathrm{~h}$ and became spheroids in
$72 \mathrm{~h}$. There was no tube formation in the CS hydrogel. In the fibrin gel, ECs formed capillary-like structures after $24 \mathrm{~h}$ (and $72 \mathrm{~h}$ ) of culture. In the CF hydrogel, ECs formed spheroids after $24 \mathrm{~h}$, and the capillary-like tubes were observed to extend from the spheroids in $72 \mathrm{~h}$. Notably, the vascular network in the CF hydrogel had a greater mean branch length and branching point density than that in fibrin gel (Figure 3c). We also observed that the CF hydrogel with varying amounts of fibrinogen/thrombin (\%) influenced the morphology and tube formation of the ECs (Supplementary Figure S3). Although an extensive network of capillary-like structures was observed for the ECs in the CF hydrogel with the fibrinogen/thrombin ratio of 0.005:0.002 (\%), poor tube formation was found in the CF hydrogel with the fibrinogen/thrombin (\%) higher or lower than 0.005:0.002 (\%). Therefore, CF hydrogel with appropriately low fibrinogen content was favorable for capillary formation. Moreover, ECs formed capillarylike structures in CF hydrogel only when the ingredients of the CS solution and fibrinogen solution were premixed before addition of the DF-PEG solution and thrombin solution, but not when the DF-PEG solution was added in a different order (CF' hydrogel) (Supplementary Figure S4). The gross observation and rheological analyses showed that the CF' hydrogel had limited self-healing capability but exhibited the injectability. Moreover, the CF' hydrogel became sol-like at a much larger strain of $>160 \%$ without the critical sol-gel state (Supplementary Figure S4). We propose that the network structure may be different in these cases, thereby influencing the capillary formation, as will be discussed later.

The RGD sequence is found in many extracellular matrix proteins and serves as the binding motif for cell surface integrin receptors. ECs seeded upon RGD-modified poly(ethylene glycol) diacrylate hydrogels have been found to show better spreading and adhesion at $24 \mathrm{~h}$ postseeding compared with those on the unmodified polymer. ${ }^{21}$ We also compared the CF hydrogel to the CS hydrogel added with RGD protein (CS-RGD hydrogel) (Supplementary Figure S5). ECs in the CS-RGD hydrogel were aggregated and became large spheroids at $72 \mathrm{~h}$ but did not exhibit tube formation. Either thrombin or fibrinogen alone has been reported to exert chemotactic effects on ECs and to upregulate the expression of the VEGF receptors (VEGFRs), thus inducing vasculogenesis in vitro. ${ }^{30,31}$ For this reason, the CS hydrogel that contained $0.002 \%$ thrombin (CS-thrombin) and the CS hydrogel that contained $0.005 \%$ fibrinogen (CS-fibrinogen) were also included for comparison (Supplementary Figure S5). However, neither hydrogel showed capillary formation. The above results indicated that RGD, thrombin and fibrinogen alone were not sufficient to induce the vasculogenesis of ECs in hydrogels.

The properties of the ECs in various hydrogels are shown in Figure 4. ECs embedded in CF hydrogel expressed a greater level of vasculogenesis marker genes (VEGFR, integrin $\alpha 4$ and endothelial nitric oxide synthase) than those in fibrin gel after 3 days (Figure $4 a$ ). The expression levels of VEGFR and endothelial nitric oxide synthase for ECs in the CS hydrogel were lower than those in fibrin gel, and the expression of integrin $\alpha 4$ was higher. These marker gene expression levels suggested that CF hydrogel is more favorable for vasculogenesis of ECs than the fibrin gel or CS hydrogel. With regard to the possible cellular mechanism, vasculogenesis is regulated by changes in the $\mathrm{O}_{2}$ tension and aerobic glycolysis. ${ }^{32}$ Previous research has indicated that ECs enhance their glycolysis, thus inducing vasculogenesis. ${ }^{33}$ Moreover, mitochondrial respiration triggers the EC apoptosis, which is associated with vessel regression, thus providing a crucial balance to EC proliferation during angiogenesis. ${ }^{33}$ We directly evaluated the metabolism (glycolysis and mitochondrial respiration) of ECs that were encapsulated in each gel by the bioenergetic assay. Analysis of the 


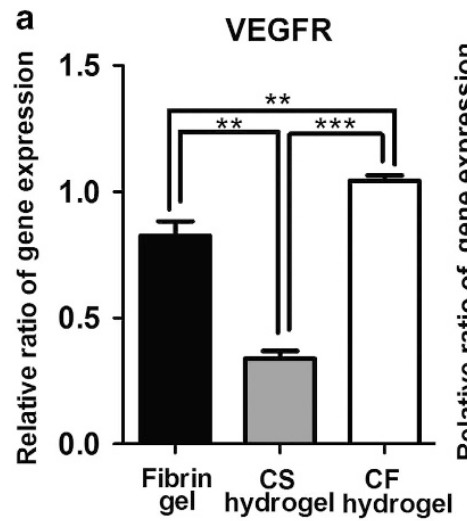

b

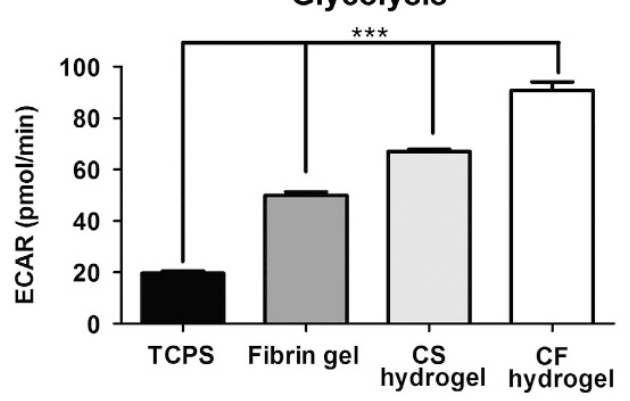

Integrin $\alpha 4$

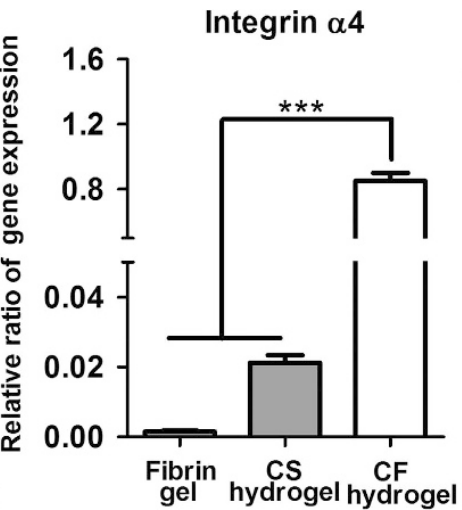

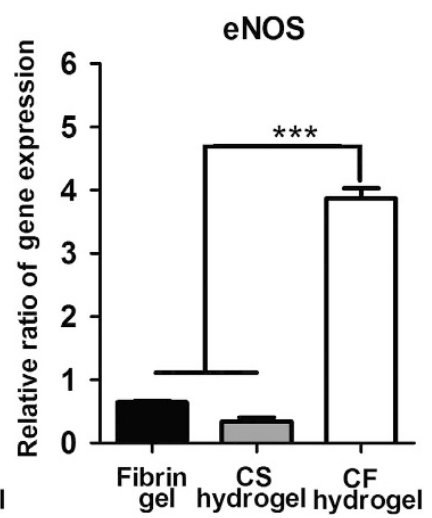

C

Mitochondrial respiration

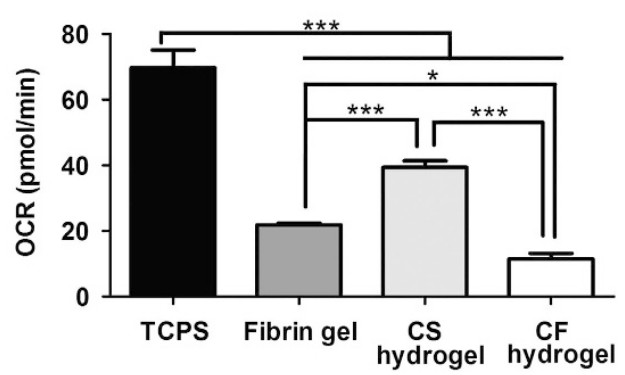

Figure 4 Behavior of vascular endothelial cells (ECs) encapsulated in different hydrogels. (a) The expression of specific vascular endothelial-related genes (VEGFR, intergrin $\alpha 4$ and endothelial nitric oxide synthase), analyzed by real-time RT-PCR for cells in gels after $72 \mathrm{~h}$. (b, c) The metabolism of ECs. In panel (b), the extracellular acidification rate was measured for ECs in different hydrogels, as an indicator of glycolysis. In panel (c), the oxygen consumption rate was measured for ECs to quantify the mitochondrial respiration in different hydrogels. ${ }^{*} P<0.05,{ }^{* *} P<0.005$, ${ }^{* * *} P<0.001$, among the indicated groups.

extracellular acidification rate with a Seahorse XF Flux Analyzer revealed the basal glycolysis levels of the cells. As shown in Figure $4 \mathrm{~b}$, the basal glycolysis measured at 3 days significantly increased for the ECs embedded in all of the gels, as compared with the tissue culture polystyrene control. Furthermore, the basal glycolysis was the highest in the ECs embedded in the CF hydrogel. The oxygen consumption rate is used as a parameter for assessing mitochondrial respiration (Figure 4c). The basal mitochondrial respiration at 3 days significantly decreased for ECs embedded in all of the gels compared with the tissue culture polystyrene control. Moreover, the basal mitochondrial respiration in ECs embedded in the CF hydrogel was lower than that of ECs embedded in the CS hydrogel and was even lower than that of ECs embedded in the fibrin gel. Together, these results suggested that ECs in the CF hydrogel may have switched their metabolism from mitochondrial respiration to glycolysis, thereby inducing vasculogenesis.

Because the in vitro evidence supported the outstanding angiogenic effect of the CF self-healing gel, we sought to confirm that finding by in vivo assays. Here we used a unique transgenic zebrafish $\mathrm{Tg}$ (flila:EGFP) in which all of the ECs showed green fluorescence because of the expression of EGFP. The Tg (flila:EGFP) zebrafish embryos are a powerful model system for an angiogenesis assay. ${ }^{24,34}$ Injection of angiogenic growth factor in the perivitelline space of zebrafish embryos induces an angiogenic response characterized by the growth of ectopic blood vessels from the subintestinal vessel basket. ${ }^{35}$ In the current study, the CS hydrogel or CF hydrogel was injected into the perivitelline space of zebrafish embryos at $24 \mathrm{hpf}$, and the pattern of the subintestinal vessels was observed at 1 day postinjection. The fibrin gel could not be injected with a needle of the same gauge, and thus it was not used in this study. The zebrafish embryos were divided into three groups ( $n=90$ per group). The first group consisted of zebrafish embryos that were injected with PBS in the perivitelline space at $24 \mathrm{hpf}$ as a blank control. The other two groups were injected with either CF hydrogel or CS hydrogel at $24 \mathrm{hpf}$. At 1 day postinjection, the pattern of subintestinal vessels was examined with a fluorescence microscope. The amount of ECs was evaluated by the expression of EGFP. The number of branching points was quantified. The latter, as mentioned above, is an index of the capillary-forming ability.

The results from the study in Tg (flila:EGFP) zebrafish embryos are shown in Figure 5. The pattern of subintestinal vessels was demonstrated by the fluorescent images (Figure 5a). Compared with that in the control (injected with PBS), the ectopic angiogenesis in the group injected with the CS hydrogel was decreased. Moreover, angiogenesis was induced in the group injected with the CF hydrogel. The EGFP gene expression in each group is shown in Figure 5b. The expression level of the EGFP gene was reduced in the group injected with the CS hydrogel and was enhanced (more than twofold) in the group injected with the CF hydrogel, compared with the control. This result indicated that the population of vascular ECs was increased by the injection of CF hydrogel and was decreased by the injection of CS hydrogel. The number of vessel branching points is shown in Figure 5c. Again, the group injected with the CS hydrogel had fewer branching points and that injected with the CF hydrogel had significantly more branching points (more than twice) vs the control. Together, these quantitative data indicated that the CF self-healing hydrogel can be injected and can induce an angiogenic response in vivo.

As mentioned earlier, CS has been widely used in the pharmaceutical and tissue engineering fields. Previous studies have suggested that 


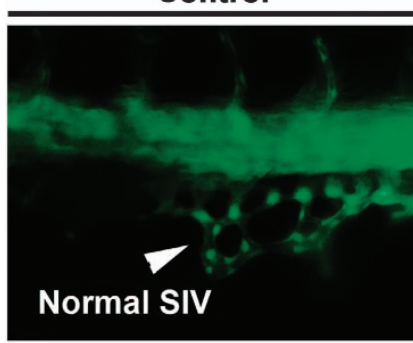

Control

b

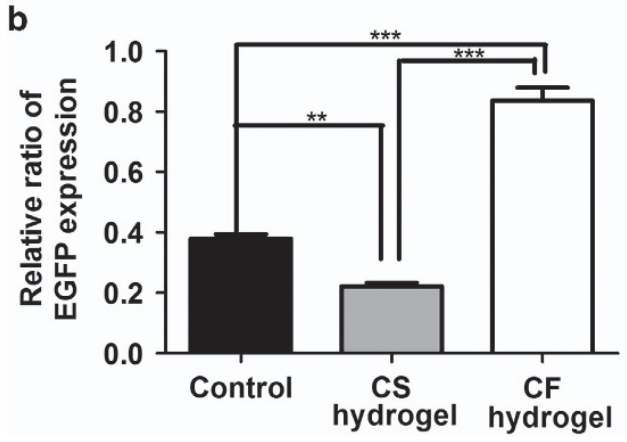

CS hydrogel

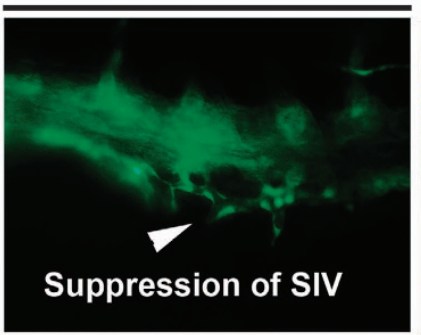

CF hydrogel

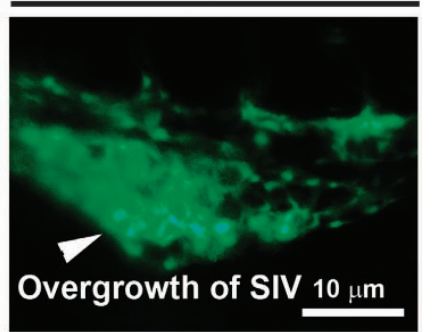

c

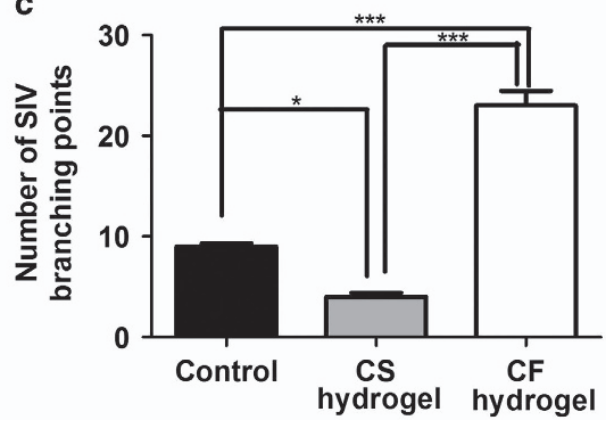

Figure 5 Injection of CF hydrogel induces blood capillary formation in vivo. (a) The pattern for the subintestinal vessels (SIV) in Tg (fli1a:EGFP) zebrafish embryos in which all of the vascular endothelial cells expressed enhanced green fluorescent protein (EGFP). Each embryo was injected with PBS, $\mathrm{CS}$ hydrogel or CF hydrogel at $24 \mathrm{~h}$ postfertilization (hpf). Only gel (not containing cells) was injected alone. The fluorescence microscopic images of the Tg (flila:EGFP) zebrafish embryos were taken at 1 day postinjection (dpi). (b) The expression of the EGFP gene analyzed by real-time RT-PCR for $\operatorname{Tg}$ (fli1a:EGFP) zebrafish embryos after 1 dpi to quantify the number of vascular endothelial cells. The expression was normalized to GAPDH. (c) The average number of SIV branching points in Tg (flila:EGFP) zebrafish embryos evaluated at $1 \mathrm{dpi}$, which is an index of the capillary-forming ability. Fibrin gel was not able to be injected through the 26-gauge needle, and thus the data are not available. ${ }^{*} P<0.05,{ }^{*} P<0.005,{ }^{* *} P<0.001$, among the indicated groups ( $n=90$ in each group).

embryonic stem cells embedded in CS hydrogel may be used to regenerate cardiomyocytes and increase the microvessel density in the infarcted heart. ${ }^{36}$ In addition, CS hydrogel may serve as a carrier for the controlled release of various angiogenic growth factors to induce neovascularization in vivo. ${ }^{16,37}$ Collagen-CS hydrogel has been found to promote the tube formation of ECs in vitro. ${ }^{24}$ Nevertheless, several studies have indicated that CS-based hydrogel does not contribute to angiogenesis in vitro and in vivo unless it is combined with ECs, stem cells or a large amount of growth factors. ${ }^{16,18,38}$ However, fibrin gel alone (without ECs or stem cells) is sufficient to induce vasculogenesis both in vitro and in vivo, ${ }^{39}$ despite its weak mechanical properties, poor injectability and potential disease transmission. CF composite films and CF nanocomposites have been prepared. ${ }^{40,41}$ Nevertheless, no previous study has developed a hybrid gel of CS and fibrin, and moreover, no study has even mentioned the self-healing capability.

We have previously synthesized an injectable, self-healing CS-based hydrogel (CS hydrogel) with a neural repair ability. ${ }^{14}$ However, the CS hydrogel did not display any angiogenic potential in the current study. The novel CF hydrogel was developed by combining CS hydrogel and a small proportion of fibrin in the gel. The results of gross observation and rheological analyses showed that the CF hydrogel possessed self-healing and injection capabilities. The self-healing process of CF hydrogel (recovery time: $\sim 6 \mathrm{~h}$ ) was even faster than that of CS hydrogel (recovery time: $\sim 12 \mathrm{~h}$ ). In addition, the strain sweep showed that CF hydrogel became sol-like at a strain of $\sim \geqslant 100 \%$, whereas the network of CS hydrogel collapsed at a greater strain of $\sim 130 \%$. The structure of the CF hydrogel was more sensitive to stress/strain than the CS hydrogel, thus possibly accounting for the faster self-healing process of the CF hydrogel. Moreover, the CF hydrogel, when subjected to a strain between 100 and $200 \%$, demonstrated close values of $G^{\prime}$ and $G^{\prime \prime}\left(G^{\prime \prime}\right.$ slightly larger than $\left.G^{\prime}\right)$. This finding was indicative of a viscoelastic sol, that is, fluid-like but with some intact structure (fast recovered structure). The interesting critical sol-gel state of CF hydrogel was not seen in the CS hydrogel. The possible underlying mechanism may be the formation of an interpenetrating polymer network (IPN) between CS and fibrin in CF hydrogel. However, the CS and CF hydrogels had similar $G^{\prime}$. Because the hydrogel network is constructed through Schiff-base transient linkages between amine groups of glycol CS or fibrinogen and benzaldehyde groups on DF-PEG termini, the polymer chains in the hydrogel network were expected to de-crosslink and re-crosslink continuously, thus resulting in the self-healing ability of the hydrogel. The faster self-healing properties of the CF hydrogel may be attributed to the increasing amount of amine groups from the fibrinogen, which in turn would promote the exchange reaction responsible for the dynamic property of the hydrogel. A faster exchange should result in a weaker hydrogel for $\mathrm{CF}$, but the presence of the interpenetrating fibrin hydrogel might compensate for this loss. This arrangement may be one of the possible explanations for the similar $G^{\prime}$ observed through rheology.

We observed very distinct extents of capillary-formation and rheological properties when the ingredients were mixed in different orders (Supplementary Figure S4). The order of mixing determined whether the IPN formed successfully. On the basis of the results, CF hydrogel formed IPN only when DF-PEG was added after CS and fibrin were premixed, as illustrated in Supplementary Figure S6. The preadded CS and fibrinogen were able to react with DF-PEG and form transient crosslinked structures, whereas thrombin transformed fibrinogen into a fibrin network (bridge), forming an interpenetrating structure that ECs were able to align with and form tubes. The thermal 
a

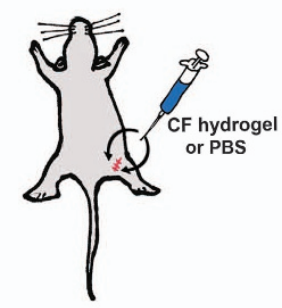

b

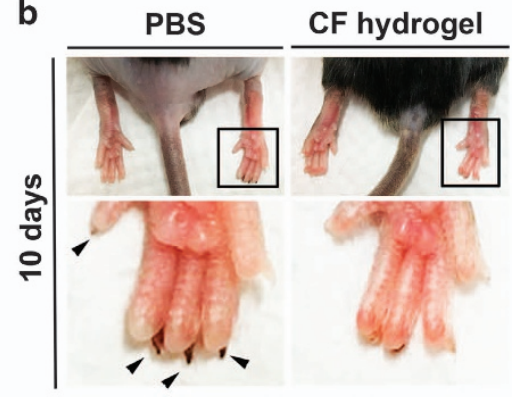

C

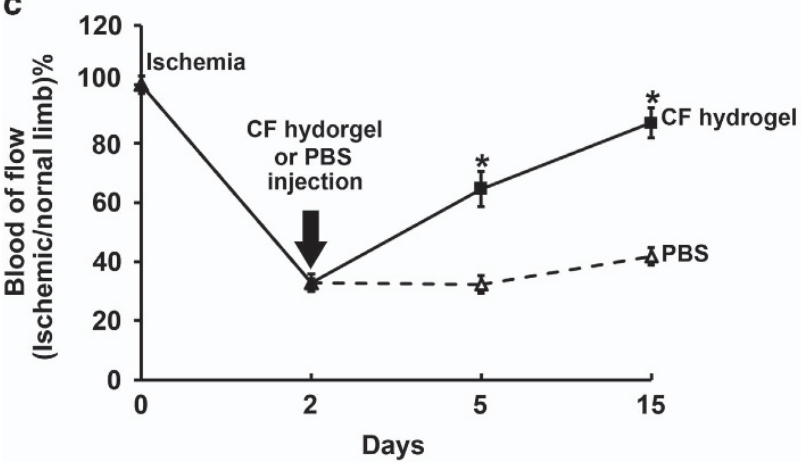

Figure 6 Injection of CF hydrogel significantly enhanced blood perfusion in the ischemic sites. (a) The left femoral artery and iliac artery in mice were ligated and then cut to induce hindlimb ischemia. The CF hydrogel or PBS (control) was intramuscularly injected into ischemic hindlimbs at 2 days postsurgery. The injections were delivered to two different sites (proximal and distal) at the ischemic area $(100 \mu \mathrm{l}$ for each site). (b) Representative images of mouse ischemic hindlimbs after injection with PBS or CF hydrogel. The PBS group experienced severe nail loss. The CF hydrogel group displayed successful limb salvage without nail loss. (c) The blood flow ratios in ischemic and healthy limbs were calculated and compared by analysis of variance Bonferroni's multiple test. The data showed a significant blood flow increase on days 5 and 15 postsurgery in the CF hydrogel-injected group $(64.7 \pm 6.0(\%)$ and $87.1 \pm 4.9(\%), n=4)$, values much higher than those in the PBS-injected group $(32.4 \pm 1.4(\%)$ and $42.9 \pm 3.0(\%), n=4),{ }^{*} P<0.05$ vs PBS-injected group. The arrow heads indicate nail loss.

stability of IPN has been found to be higher than the respective homopolymer. ${ }^{42}$ As shown in Supplementary Figure S6b, the CF' hydrogel exhibited a heterogeneous structure and phase separation. The phase separation in the CF' hydrogel may have resulted in the hindered self-healing behavior. Formation of IPN in the CF hydrogel but not the CF' hydrogel was further confirmed by thermal analyses. According to thermogravimetric analysis curves of the hydrogels (Supplementary Figure S6c), the thermal stability of the CF hydrogel was better than that of the CS and CF' hydrogels. The differential scanning calorimetry profiles (Supplementary Figure S6d) showed that the glass transition temperature $\left(T_{\mathrm{g}}\right)$ of the CS, CF and CF' hydrogels was $-53.9,-51.0$ and $-52.2^{\circ} \mathrm{C}$, respectively. The $T_{\mathrm{g}}$ of the CF hydrogel increased, compared with that of CS or CF' hydrogel. Three melting peaks of the DF-PEG segments in the CS hydrogel were observed at $21.4\left(T_{\mathrm{m} 1}\right), 55.8\left(T_{\mathrm{m} 2}\right)$ and $60.0^{\circ} \mathrm{C}\left(T_{\mathrm{m} 3}\right)$, with the latter two being associated with crystalline DF-PEG. The endothermic peak area for $T_{\mathrm{m} 2}$ and $T_{\mathrm{m} 3}$ in the CF hydrogel was significantly smaller than that in the CS and CF' hydrogels, whereas the peak area for $T_{\mathrm{m} 1}$ was much larger. Because IPN may decrease chain mobility, ${ }^{43}$ the decreased crystallinity of the DF-PEG segments in the CF hydrogel may be ascribed to the formation of the IPN structure in the hydrogel.

The suppression of crystalline DF-PEG segments in the CF hydrogel was also consistent with the more stress/strain-sensitive network of this hydrogel, as demonstrated by rheology.

The transient interpenetration of such a network may explain the critical sol-gel state of the CF hydrogel after gel deformation. Although some studies have indicated that IPN formation enhances the performance of the hydrogels, ${ }^{44-46}$ few studies have reported the influence of the IPN structure on self-healing of the hydrogel. One recent study has reported that the IPN structure improves the self-healing capability of polymer films. ${ }^{47}$ Another very recent study has prepared IPN self-healing hydrogel with various moduli $(\sim 2-10 \mathrm{kPa}) .{ }^{48}$ No study has evaluated the physiological function of IPN self-healing hydrogels in vitro and in vivo. The versatility of IPN and the steric constraints of such a network may explain the better self-healing capability of CF hydrogel after deformation. Moreover, the fibrin gel degrades fairly quickly, but the degradation rate of the fibrin-containing CF hydrogel is even slightly slower than that of the CS hydrogel. Gel degradation may be retarded by the IPN structure of the hydrogel.

In vitro, ECs in CF hydrogel displayed better angiogenic capacities than those of fibrin gel (Figure 3c). Previous studies have suggested that the glycosaminoglycans of CS form insoluble complexes with stromal cell-derived factor-1, fibroblast growth factors and plateletderived growth factors, which triggered EC aggregation and proliferation. ${ }^{49-51}$ We thus assumed that CS would trigger EC aggregation, whereas fibrin would trigger EC tube formation in our CF hydrogel. The normal level of fibrinogen is approximately $0.0015-0.0045 \%$ in the human circulatory system, and a higher risk of cardiovascular disease is related to a higher level of fibrinogen in clinical studies. ${ }^{52,53}$ The amount of fibrinogen in the CF self-healing hydrogel was similar to that in the human blood, thus potentially avoiding side effects in humans. Furthermore, thrombin alone has been shown to induce angiogenesis in vitro. ${ }^{54}$ In the current study, the addition of thrombin alone in CS hydrogel did not induce the vasculogenesis of ECs. The crosslinked network of fibrin polymers is thus important for vasculogenesis.

Zebrafish have emerged as an excellent vertebrate model system for studying vascular development and drug screening. ${ }^{24,34,35}$ In this study, the injection of CF hydrogel in the perivitelline space of zebrafish embryos induced angiogenesis. Although the zebrafish model is a convenient tool to confirm the in vivo angiogenesis induced via CF hydrogel, the therapeutic potential remains to be verified by translational studies. Here CF hydrogel was preliminarily tested in the disease model of mouse hindlimb ischemia, which represents peripheral arterial occlusive diseases. The recovery rate of blood flow in ischemic mice is depicted in Figures 6a-c. Single-dose local injection of CF hydrogel in 2-day ischemic mice demonstrated a rescue of blood flow at 5 and 15 days, in comparison with that in the control group (PBS-injected mice) (Figures $6 \mathrm{~b}$ and c). Long-term results of injection in chronic (15-day) ischemic mice are included in Supplementary Figure S7. The results suggested that CF hydrogel can rescue the function of an impaired blood circulatory system in acute and chronic ischemic diseases. However, more extensive studies should be performed to support the clinical potential of this hydrogel.

Angiogenesis is a normal and essential process in growth and development as well as in wound healing. Unfortunately, it is also a fundamental step for the transition of tumors from a benign state to a malignant state. ${ }^{55}$ In both normal and abnormal tissues, the EC metabolism is switched from mitochondrial respiration to glycolysis, thereby inducing angiogenesis. ${ }^{55}$ There are thus potential and unanticipated cancer risks after the implantation of angiogenic 
scaffolds. ${ }^{56}$ In the current study, glycolysis was increased and mitochondrial respiration decreased after ECs were embedded in CF hydrogel. Although the CS self-healing gel also increased glycolysis, it decreased mitochondrial respiration to a lesser degree than did the fibrin gel or CF hydrogel. Recent investigations have revealed that CS and its derivatives exhibit antitumor activity in both in vitro and in vivo models by promoting cells to secrete cytokines. ${ }^{57,58}$ Our zebrafish experiment also demonstrated an antiangiogenic effect of the CS self-healing hydrogel. We hope that the composite CF hydrogel will be able to retain the antitumor effect of CS and induce angiogenesis without a risk of causing cancer.

\section{CONCLUSION}

A novel CF-based injectable and self-healing hydrogel was established in this study. The IPN formation in the hydrogel was associated with the excellent self-healing and capillary-inducing capacities of the hydrogel. This study provides insight regarding the effects of the gel network on the self-healing and biomimetic properties of a hydrogel. We propose that other extracellular matrix proteins may also form a self-healing interpenetrating hybrid gel with a CS-based self-healing hydrogel, thus presenting various self-healing possibilities and degradation features for a variety of different biomedical applications.

\section{CONFLICT OF INTEREST}

The authors declare no conflict of interest.

\section{ACKNOWLEDGEMENTS}

This research was supported by the Program for Additive Manufacturing (MOST 105-2811-E-002-007), Ministry of Science and Technology, Taiwan, R.O.C, the University Cutting-Edge Steering Research Project (NTU-CESRP-103R104943), International Collaboration Research Project (NTU-ICRP-105R7564) and the National Health Research Institutes (CS-106-PP-15). We also thank the Taiwan Zebrafish Core Facility at National Taiwan University (NTU-ERP-104R8600) for their facilities and technical support.

1 Lee, K. Y. \& Mooney, D. J. Hydrogels for tissue engineering. Chem. Rev. 101 1869-1879 (2001).

2 Lovett, M., Lee, K., Edwards, A. \& Kaplan, D. L. Vascularization strategies for tissue engineering. Tissue Eng. B Rev. 15, 353-370 (2009).

3 Yu, L. \& Ding, J. Injectable hydrogels as unique biomedical materials. Chem. Soc. Rev. 37, 1473-1481 (2008)

4 Annabi, N., Tamayol, A., Uquillas, J. A., Akbari, M., Bertassoni, L. E., Cha, C., CamciUnal, G., Dokmeci, M. R., Peppas, N. A. \& Khademhosseini, A. 25th anniversary article: Rational design and applications of hydrogels in regenerative medicine. Adv. Mater. 26, 85-123 (2014).

5 Klouda, L. \& Mikos, A. G. Thermoresponsive hydrogels in biomedical applications. Eur. J. Pharmaceut. Biopharmaceut. 68, 34-45 (2008).

6 Hager, M. D., Greil, P., Leyens, C., van der Zwaag, S. \& Schubert, U. S. Self-healing materials. Adv. Mater. 22, 5424-5430 (2010).

7 Casuso, P., Perez-San Vicente, A., Iribar, H., Gutierrez-Rivera, A., Izeta, A., Loinaz, I., Cabanero, G., Grande, H. J., Odriozola, I. \& Dupin, D. Aurophilically cross-linked "dynamic" hydrogels mimicking healthy synovial fluid properties. Chem. Commun. 50, 15199-15201 (2014).

8 Barcan, G. A., Zhang, X. \& Waymouth, R. M. Structurally dynamic hydrogels derived from 1,2-dithiolanes. J. Am. Chem. Soc. 137, 5650-5653 (2015).

9 Casuso, P., Odriozola, I., Perez-San Vicente, A., Loinaz, I., Cabanero, G., Grande, H. J. \& Dupin, D. Injectable and self-healing dynamic hydrogels based on metal(I)-thiolate/ disulfide exchange as biomaterials with tunable mechanical properties. Biomacromolecules 16, 3552-3561 (2015).

10 Yuan, Y. C., Yin, T., Rong, M. Z. \& Zhang, M. Q. Self healing in polymers and polymer composites. Concepts, realization and outlook: A review. Express. Polym. Lett. 2, 238-250 (2008).

11 Zhang, Y., Yang, B., Zhang, X., Xu, L., Tao, L., Li, S. \& Wei, Y. A magnetic self-healing hydrogel. Chem. Commun. 48, 9305-9307 (2012).

12 Yan, Q., Yuan, J., Zhang, F., Sui, X., Xie, X., Yin, Y., Wang, S. \& Wei, Y. Cellulose-based dual graft molecular brushes as potential drug nanocarriers: stimulus-responsive micelles, self-assembled phase transition behavior, and tunable crystalline morphologies. Biomacromolecules 10, 2033-2042 (2009).

13 Cao, L., Cao, B., Lu, C., Wang, G., Yu, L. \& Ding, J. An injectable hydrogel formed by in situ cross-linking of glycol chitosan and multi-benzaldehyde functionalized PEG analogues for cartilage tissue engineering. J. Mater. Chem. 3, 1268-1280 (2015).

14 Tseng, T. C., Tao, L., Hsieh, F. Y., Wei, Y., Chiu, I. M. \& Hsu, S. H. An injectable, selfhealing hydrogel to repair the central nervous system. Adv. Mater. 27, 3518-3524 (2015).

15 Kumar, V. A., Taylor, N. L., Shi, S., Wang, B. K., Jalan, A. A., Kang, M. K., Wickremasinghe, N. C. \& Hartgerink, J. D. Highly angiogenic peptide nanofibers. ACS Nano 9, 860-868 (2015).

16 Fujita, M., Ishihara, M., Simizu, M., Obara, K., Ishizuka, T., Saito, Y., Yura, H., Morimoto, Y., Takase, B., Matsui, T., Kikuchi, M. \& Maehara, T. Vascularization in vivo caused by the controlled release of fibroblast growth factor-2 from an injectable chitosan/non-anticoagulant heparin hydrogel. Biomaterials 25, 699-706 (2004).

17 Rafat, M., Li, F., Fagerholm, P., Lagali, N. S., Watsky, M. A., Munger, R., Matsuura, T. \& Griffith, M. PEG-stabilized carbodiimide crosslinked collagen-chitosan hydrogels for corneal tissue engineering. Biomaterials 29, 3960-3972 (2008).

18 Ahmadi, R., Burns, A. J. \& de Bruijn, J. D. Chitosan-based hydrogels do not induce angiogenesis. J. Tissue Eng. Regen. Med. 4, 309-315 (2010).

19 Shaikh, F. M., Callanan, A., Kavanagh, E. G., Burke, P. E., Grace, P. A. \& McGloughlin, T. M. Fibrin: a natural biodegradable scaffold in vascular tissue engineering. Cells Tissues Organs 188, 333-346 (2008).

20 Zhang, Y., Tao, L., Li, S. \& Wei, Y. Synthesis of multiresponsive and dynamic chitosanbased hydrogels for controlled release of bioactive molecules. Biomacromolecules 12 , 2894-2901 (2011)

21 Chang, J. C., Hsu, S. H. \& Chen, D. C. The promotion of chondrogenesis in adiposederived adult stem cells by an RGD-chimeric protein in 3D alginate culture. Biomaterials 30, 6265-6275 (2009).

22 Hagiwara, H., Shimonaka, M., Morisaki, M., Ikekawa, N. \& Inada, Y. Sitosterolstimulative production of plasminogen activator in cultured endothelial cells from bovine carotid artery. Thromb. Res. 33, 363-370 (1984).

23 Kimmel, C. B., Ballard, W. W., Kimmel, S. R., Ullmann, B. \& Schilling, T. F. Stages of embryonic development of the zebrafish. Dev. Dynam. 203, 253-310 (1995).

24 Nicoli, S. \& Presta, M. The zebrafish/tumor xenograft angiogenesis assay. Nat. Protoc. 2, 2918-2923 (2007).

25 Niiyama, H., Huang, N. F., Rollins, M. D. \& Cooke, J. P. Murine model of hindlimb ischemia. J. Vis. Exp. 23, 1305 (2009).

26 Kniazeva, E. \& Putnam, A. J. Endothelial cell traction and ECM density influence both capillary morphogenesis and maintenance in 3-D. Am. J. Physiol. Cell Physiol. 297, C179-C187 (2009)

27 Krishnan, L., Hoying, J. B., Nguyen, H., Song, H. \& Weiss, J. A. Interaction of angiogenic microvessels with the extracellular matrix. Am. J. Physiol. 293, H3650-H3658 (2007).

28 Kniazeva, E., Kachgal, S. \& Putnam, A. J. Effects of extracellular matrix density and mesenchymal stem cells on neovascularization in vivo. Tissue Eng. A 17, 905-914 (2011).

29 Wu, W., Allen, R. A. \& Wang, Y. Fast-degrading elastomer enables rapid remodeling of a cell-free synthetic graft into a neoartery. Nat. Med. 18, 1148-1153 (2012)

30 Maragoudakis, M. E., Tsopanoglou, N. E. \& Andriopoulou, P. Mechanism of thrombininduced angiogenesis. Biochem. Soc. Trans. 30, 173-177 (2002).

31 Shiose, S., Hata, Y., Noda, Sassa, Y., Takeda, A., Yoshikawa, H., Fujisawa, K., Kubota, T. \& Ishibashi, T. Fibrinogen stimulates in vitro angiogenesis by choroidal endothelial cells via autocrine VEGF. Graefes Arch. Clin. Exp. Ophthalmol. 242, 777-783 (2004).

32 Fraisl, P., Mazzone, M., Schmidt, T. \& Carmeliet, P. Regulation of angiogenesis by oxygen and metabolism. Dev. Cell 16, 167-179 (2009).

33 Stapor, P., Wang, X., Goveia, J., Moens, S. \& Carmeliet, P. Angiogenesis revisited- role and therapeutic potential of targeting endothelial metabolism. J. Cell Sci. 127, 4331-4341 (2014)

34 Lawson, N. D. \& Weinstein, B. M. In vivo imaging of embryonic vascular development using transgenic zebrafish. Dev. Biol. 248, 307-318 (2002).

35 Nicoli, S., De Sena, G. \& Presta, M. Fibroblast growth factor 2 -induced angiogenesis in zebrafish: the zebrafish yolk membrane (ZFYM) angiogenesis assay. J. Cell. Mol. Med. 13, 2061-2068 (2009).

36 Lu, W. N., Lu, S. H., Wang, H. B., Li, D. X., Duan, C. M., Liu, Z. Q., Hao, T., He, W. J., Xu, B., Fu, Q., Song, Y. C., Xie, X. H. \& Wang, C. Y. Functional improvement of infarcted heart by co-injection of embryonic stem cells with temperature-responsive chitosan hydrogel. Tissue Eng. A 15, 1437-1447 (2009).

37 Yeo, Y., Geng, W., Ito, T., Kohane, D. S., Burdick, J. A. \& Radisic, M. Photocrosslinkable hydrogel for myocyte cell culture and injection. J. Biomed. Mater. Res. B Appl. Biomater. 81, 312-322 (2007).

38 Deng, C., Zhang, P., Vulesevic, B., Kuraitis, D., Li, F., Yang, A. F., Griffith, M., Ruel, M. \& Suuronen, E. J. A collagen-chitosan hydrogel for endothelial differentiation and angiogenesis. Tissue Eng. A 16, 3099-3109 (2010).

39 Jockenhoevel, S., Zund, G., Hoerstrup, S. P., Chalabi, K., Sachweh, J. S., Demircan, L., Messmer, B. J. \& Turina, M. Fibrin gel-advantages of a new scaffold in cardiovascular tissue engineering. Eur. J. Cardiothorac. Surg. 19, 424-430 (2001).

40 Pandima Devi, M., Sastry, T. P. \& Meignanalakshmi, S. Preparation and characterization of fibrin-chitosan composite and its in vivo studies. IOSR J. Pharm. 2, 21-32 (2012). 
41 Zhou, W., Zhao, M., Zhao, Y. \& Mou, Y. A fibrin gel loaded with chitosan nanoparticles for local delivery of rhEGF: preparation and in vitro release studies. J. Mater. Sci. Mater. Med. 22, 1221-1230 (2011).

42 Thomas, S., Grande, D., Cvelbar, U., Raju, K. V. S. N., Narayan, R., Thomas, S. \& Akhina, H. Micro- and Nano-Structured Interpenetrating Polymer Networks: From Design to Applications (John Wiley \& Sons, Inc., Hoboken, NJ, USA, 2016).

43 Jacob, J., Klepac, D., Didović, D., Sandesh, C. J., Liu, Y., Raju, K. V. S. N., Pius, A., Valić, S. \& Thomas, S. Main chain and segmental dynamics of semi interpenetrating polymer networks based on polyisoprene and poly(methyl methacrylate). Polymer 51, 2390-2402 (2010).

44 Suresh, P. K., Suryawani, S. K. \& Dewangan, D. Chitosan based interpenetrating polymer network (IPN) hydrogels: a potential multicomponent oral drug delivery vehicle. Pharmacie Globale Int. J. Compr. Pharm. 8, 1-8 (2011).

45 Kulkarni, P. V. \& Keshavayya, J. Chitosan sodium alginate biodegradable interpenetrating polymer network (IPN) beads for delivery of ofloxacin hydrochloride. Int. J. Pharm. Pharmaceut. Sci. 2, 77-82 (2010).

46 Rowe, S. L. \& Stegemann, J. P. Interpenetrating collagen-fibrin composite matrices with varying protein contents and ratios. Biomacromolecules 7, 2942-2948 (2006).

47 Peterson, A. M., Kotthapalli, H., Rahmathullah, M. A. M. \& Palmese, G. R. Investigation of interpenetrating polymer networks for self-healing applications. Compos. Sci. Technol. 72, 330-336 (2012).

48 Rodell, C. B., Dusaj, N. N., Highley, C. B. \& Burdick, J. A. Injectable and cytocompatible tough double-network hydrogels through tandem supramolecular and covalent crosslinking. Adv. Mater. 28, 8419-8424 (2016).

49 Denuziere, A., Ferrier, D., Damour, O. \& Domard, A. Chitosan-chondroitin sulfate and chitosan-hyaluronate polyelectrolyte complexes: biological properties. Biomaterials 19 , 1275-1285 (1998).

50 Mbemba, E., Gluckman, J. C. \& Gattegno, L. Glycan and glycosaminoglycan binding properties of stromal cell-derived factor (SDF)-lalpha. Glycobiology 10, 21-29 (2000).

51 Ornitz, D. M. FGFs, heparan sulfate and FGFRs: complex interactions essential for development. Bioessays 22, 108-112 (2000).
52 Page, R. C. \& Schroeder, H. E. Pathogenesis of inflammatory periodontal disease: a summary of current work. Lab. Invest. 34, 235-249 (1976).

53 Salvi, V. Medical and Surgical Diagnostic Disorders in Pregnancy (Jaypee Brothers Publishers, New Delhi, India, 2003)

54 Hua, Y., Keep, R. F., Gu, Y. \& Xi, G. Thrombin and brain recovery after intracerebral hemorrhage. Stroke 40, S88-S89 (2009).

55 Wallace, D. C. Mitochondria and cancer. Nat. Rev. Cancer 12, 685-698 (2012).

56 Li, R. K. \& Weisel, R. D. Cardiac Regeneration and Repair (Elsevier, Oxford, UK, 2014).

57 Maeda, Y. \& Kimura, Y. Antitumor effects of various low-molecular-weight chitosans are due to increased natural killer activity of intestinal intraepithelial lymphocytes in sarcoma 180-bearing mice. J. Nutr. 134, 945-950 (2004).

58 Harish Prashanth, K. V. \& Tharanathan, R. N. Depolymerized products of chitosan as potent inhibitors of tumor-induced angiogenesis. Biochim. Biophys. Acta 1722, 22-29 (2005)

(c) This work is licensed under a Creative Commons Attribution 4.0 International License. The images or other third party material in this article are included in the article's Creative Commons license, unless indicated otherwise in the credit line; if the material is not included under the Creative Commons license, users will need to obtain permission from the license holder to reproduce the material. To view a copy of this license, visit http:// creativecommons.org/licenses/by/4.0/

(C) The Author(s) 2017

Supplementary Information accompanies the paper on the NPG Asia Materials website (http://www.nature.com/am) 